\title{
Vertebral Metastasis Treated by Vertebroplasty, a Cause of Respiratory Failure: Case Report and Literature Review
}

\author{
Mathieu Chevallier ${ }^{a} \quad$ Chloé Chevallier-Lugon ${ }^{b} \quad$ Alex Friedlaender $^{a}$ \\ Alfredo Addeo ${ }^{a}$ \\ aDepartment of Oncology, University Hospitals of Geneva, Geneva, Switzerland; \\ bepartment of Primary Care Medicine, University Hospitals of Geneva, Geneva, \\ Switzerland
}

\author{
Keywords \\ Bone metastasis · Lung cancer · Vertebroplasty
}

\section{Abstract}

Bone is a frequent site of metastases in advanced cancers including lung, breast, prostate, kidney, or myeloma. Lesions are commonly located on the spine. Neoplastic invasion of the vertebral body can result in painful vertebral fractures, leading to disability and substantial morbidity. Percutaneous vertebroplasty is a minimally invasive surgical procedure used to treat spinal fractures due to osteolytic tumors. It could result in pain reduction or resolution in $80-90 \%$ of patients with fractures, and it improves stability. Although considered safe, vertebroplasty has been associated over the years with life-threatening complications. We have reported the case of a 55-year-old patient with lung adenocarcinoma, who underwent vertebroplasty for a pathological neoplastic fracture of $\mathrm{L} 2$. The procedure was complicated by a leak of cement into the systemic venous circulation, characterized by an $11-\mathrm{cm}$ filament in the right heart chambers and multiple pulmonary emboli. To our knowledge, only one similar case was previously reported, involving an intracardiac cement filament longer than $10 \mathrm{~cm}$. The data are scant, hence the importance of collecting and reporting possible complications about what is perceived as a rather safe procedure. The case highlights the need for a robust postprocedure imaging plan to detect complications, which can impact patients' morbidity and survival. 


\section{Introduction}

Bone is a frequent site of metastases in advanced cancers including lung, breast, prostate, kidney or myeloma. Lesions are commonly located on the spine. Neoplastic invasion of the vertebral body can result in painful vertebral fractures, leading to disability and substantial morbidity [1]. Percutaneous vertebroplasty is a minimally invasive surgical procedure used to treat vertebral hemangiomas and spinal fractures due to osteoporosis or osteolytic tumors of the spine. This method leads to pain reduction or resolution in $80-90 \%$ of patients with fractures [2], and it improves stability. Generally considered safe [3], vertebroplasty has been associated over the years with life-threatening complications (i.e., compressive myelopathy and radiculopathy due to cement leakage) [4].

Here, we describe the case of a 55-year-old patient with lung adenocarcinoma, who underwent vertebroplasty for a pathological neoplastic fracture of L2. The procedure was complicated by a leak of cement into the systemic venous circulation, characterized by an $11-\mathrm{cm}$ filament in the right heart chambers and multiple pulmonary emboli. As cement is a friable material, the foreign body could not be removed in a secure manner, resulting in further migration into both pulmonary arteries. To our knowledge, only one similar case was previously reported, involving an intracardiac cement filament longer than $10 \mathrm{~cm}$ [5]. Data in the literature are scant, hence the importance of collecting and reporting possible complications about what is perceived as a rather safe procedure.

The case highlights the need for a robust postprocedure imaging plan to detect complications, which can impact patients' morbidity and survival.

\section{Case Report}

A 55-year-old female patient suffering from lung adenocarcinoma with a concomitant EGFR and KRAS mutation with metastatic bone and brain lesions was referred to our Oncology Department to undergo whole-brain radiotherapy. Given the nature of the mutations, systemic therapy was less likely to be highly effective [6-8]. Her medical history was also notable for vertebral metastases requiring L2 percutaneous cement vertebroplasty 1 month prior to admission. Furthermore, she suffered from Trousseau syndrome requiring anticoagulation with rivaroxaban. Upon admission, the physical examination revealed signs of respiratory distress. The chest X-ray showed a right lung infiltrate compatible with multilobar pneumonia, and revealed a dense, thread-like structure, facing the right mediastinum.

Low-dose chest computed tomography (CT) scan was then performed, confirming the presence of an 11-cm foreign body of metallic density in the right ventricle, with a 5 -cm extension in the right atrium (Fig. 1). The absence of these abnormalities on the CT scan prior to the vertebroplasty led us to the conclusion of cement embolization into the right heart chambers.

Reviewing the vertebroplasty report revealed the occurrence of minimal cement leakage into the paravertebral veins during the procedure. During hospitalization, the patient presented with sinus tachycardia, suggesting the presence of cement in the right atrium was a trigger for arrhythmia.

Thereafter, we failed to perform endovascular removal of the foreign object (Fig. 2), which was further complicated by migration of cement into the left pulmonary artery. Given the grim oncological prognosis of the patient, we decided to give up further invasive procedures and leave the cement in place. The numerous cement emboli remained throughout the hospitalization despite anticoagulation with rivaroxaban. Due to a new pulmonary embolism in the left pulmonary artery, the anticoagulation was changed to subcutaneous low-molecularweight heparin. Unfortunately, our patient died a few weeks later of respiratory failure.

\section{Karger'}


Fig. 1. Chest low-dose CT scan showing a foreign body of metallic density (arrow).

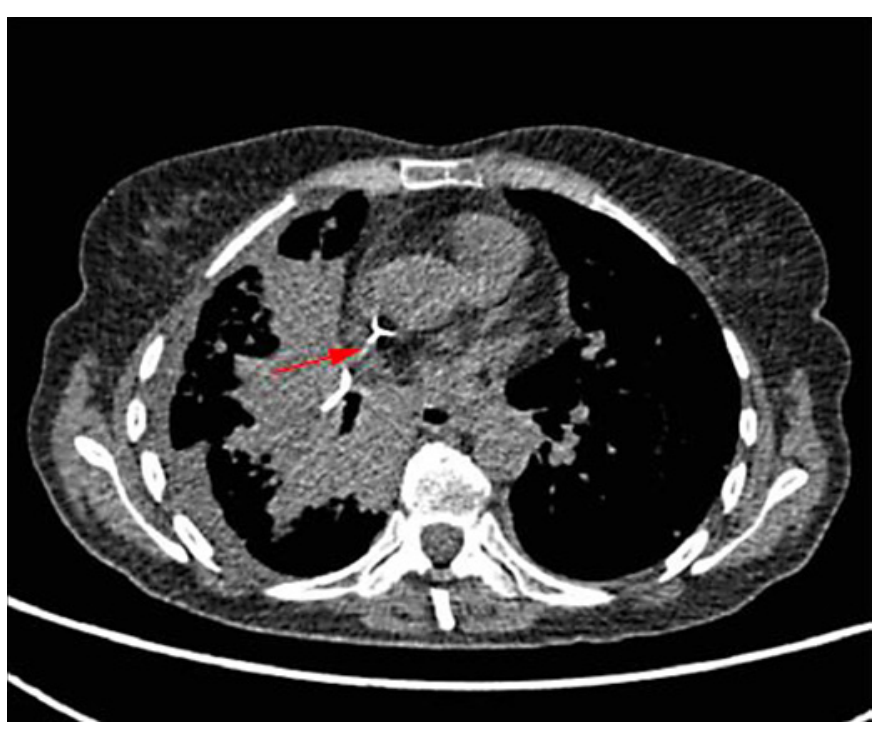

Fig. 2. Angioscopic view showing the fragmentation of the thread-like structure in the right ventricle, which then migrates to the left pulmonary artery.

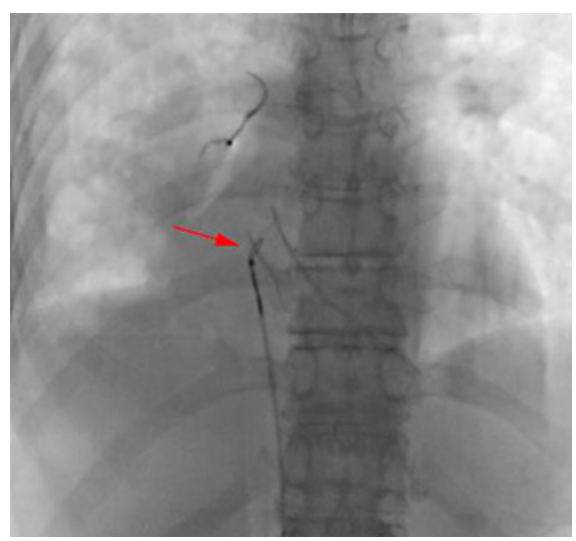

\section{Discussion}

Pathological fractures represent a significant proportion of vertebral fractures. Moreover, $70 \%$ of women with breast cancer, $70 \%$ of men with prostate cancer, and $40 \%$ of patients with lung cancer will develop bone metastases during the course of their disease [9].

Percutaneous vertebroplasty was described in 1987 by Galibert and colleagues as a minimally invasive treatment of hemangiomas. The use of vertebroplasty has since then expanded to include treatment of osteoporosis, pathological fractures related to spinal metastasis, and more rarely of traumatic fractures. Vertebroplasty for malignancy can be used as adjunctive therapy along with radiotherapy or chemotherapy. It is performed by interventional radiology specialists to stabilize vertebral fractures at risk for collapse or spinal cord compression.

This technique proved effective in the relief of pain related to metastatic fractures, and its indication for analgesic treatment of vertebral compression is widely recognized [10]. A similar approach with kyphoplasty, also corresponding to percutaneous injection of acrylic bone cement into the fracture, is possible. The difference between the two techniques is that kyphoplasty uses the inflation of a balloon in the vertebral body to create space before injecting the cement, to address curvature of the spine to restore height.

Studies showed that patients with pathological vertebral fractures who were treated with vertebroplasty or kyphoplasty had a better functional outcome at 1 month follow-up 
than patients who received non-interventional management. They also demonstrated a marked reduction in back pain and improvement in quality of life, with fewer patients using pain medications. Improvement in functional status, quality of life, and pain persisted until 1 year after the procedure. Because of the limited improvement in patients with a conservative treatment, some investigators suggest that vertebroplasty should be considered as an early treatment option for patients with cancer who have symptomatic vertebral fractures [11]. Defining exact patient selection criteria is difficult due to a lack of consensus in the literature, but trials have shown better outcomes when vertebroplasty occurred within 6 weeks of sustaining a fracture for patients with severe pain and immobility [12].

There is an apparent increase in complication rates for vertebroplasty in cancer patients. The suggested reasons include the loss of cortical integrity and tumor angiogenesis. In the current literature, few cases are found assessing adverse outcomes related to the procedure. Most reports describe the occurrence of cement pulmonary emboli. Other complications of vertebroplasty include cement leakage in paravertebral veins, infection, and leaking in the dura. Our case is, to our knowledge, the first description of the finding of cement in the heart chambers, leading to cardiac arrhythmia which can be explained by irritation of the atrium. Furthermore, we outline that cement, when in contact with blood at $37^{\circ} \mathrm{C}$, becomes filamentous and friable, making its removal challenging.

\section{Conclusion}

Vertebroplasty is a minimally invasive technique, proven to be effective to ease pain and improve quality of life in patients with vertebral metastases, with an overall low rate of complications. However, complications are possible, and some of them, such as cement embolization, can affect patients' prognosis.

Our case report prompts us to consider this risk when weighing the pros and cons of the procedure. Moreover, it suggests the benefit of monitoring patients with evidence of periprocedural cement leakage as a key to avoid further complications.

\section{Statement of Ethics}

The patient has provided written consent to disclose the case and the pictures.

\section{Conflict of Interest Statement}

The authors have no conflict of interest to disclose.

\section{Funding Sources}

Not funding was released or obtained for the present article.

\section{Author Contributions}

All the authors contributed equally to the manuscript.

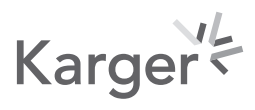




\section{References}

1 Friedlaender A, Liu SV, Passaro A, Metro G, Banna G, Addeo A. The role of performance status in small-cell lung cancer in the era of immune checkpoint inhibitors. Clin Lung Cancer. 2020 Nov;21(6):e539-e543.

2 Ali Shaibani SA, Bhatt H, editors. Vertebroplasty and kyphoplasty for the palliation of pain. Semin Interv Radiol. Stuttgart: Thieme; 2007.

3 Chew C, Craig L, Edwards R, Moss J, O’Dwyer PJ. Safety and efficacy of percutaneous vertebroplasty in malignancy: a systematic review. Clin Radiol. 2011;66(1):63-72.

4 Bròdano GB, Cappuccio M, Gasbarrini A, Bandiera S, De Salvo F, Cosco F, et al . Vertebroplasty in the treatment of vertebral metastases: clinical cases and review of the literature. Eur Rev Med Pharmacol Sci. 2007;11(2): 91.

5 Makary MS, Zucker IL, Sturgeon JM. Venous extravasation and polymethylmethacrylate pulmonary embolism following fluoroscopy-guided percutaneous vertebroplasty. Acta Radiol Open. 2015;4(8):2058460115595660.

6 Chevallier M, Tsantoulis P, Addeo A, Friedlaender A. Influence of concurrent mutations on overall survival in EGFR-mutated non-small cell lung cancer. Cancer Genomics Proteomics. 2020;17(5):597-603.

7 Friedlaender A, Drilon A, Weiss GJ, Banna GL, Addeo A. KRAS as a druggable target in NSCLC: Rising like a phoenix after decades of development failures. Cancer Treat Rev. 2020;85:101978.

8 Torralvo J, Friedlaender A, Achard V, Addeo A. The activity of immune checkpoint inhibition in KRAS mutated non-small cell lung cancer: a single centre experience. Cancer Genomics Proteomics. 2019 Nov-Dec;16(6): 577-82.

9 Macedo F, Ladeira K, Pinho F, Saraiva N, Bonito N, Pinto L, et al . Bone metastases: an overview. Oncol Rev. 2017;11(1):321.

10 Klazen CA, Lohle PN, de Vries J, Jansen FH, Tielbeek AV, Blonk MC, et al . Vertebroplasty versus conservative treatment in acute osteoporotic vertebral compression fractures (Vertos II): an open-label randomised trial. Lancet. 2010;376(9746):1085-92.

11 Berenson J, Pflugmacher R, Jarzem P, Zonder J, Schechtman K, Tillman JB, et al . Balloon kyphoplasty versus non-surgical fracture management for treatment of painful vertebral body compression fractures in patients with cancer: a multicentre, randomised controlled trial. Lancet Oncol. 2011;12(3):225-35.

12 Montagu A, Speirs A, Baldock J, Corbett J, Gosney M. A review of vertebroplasty for osteoporotic and malignant vertebral compression fractures. Age Ageing. 2012;41(4):450-5. 